\title{
Weekly Assessment of Growth Performance of Broilers Fed Diet Supplemented with Garlic and Turmeric Powder and their Combination
}

\author{
Khwairakpam Ratika ${ }^{1}$, R.K. James $\operatorname{Singh}^{2}$, Ram Kumar Singh ${ }^{1}$ and Mala Singh ${ }^{3}$ \\ ${ }^{1}$ Division of Animal Nutrition, ${ }^{3}$ Division of Animal Physiology, ICAR- National Dairy \\ Research Institute, Karnal-132001- India \\ ${ }^{2}$ Division of Veterinary Biotechnology, ICAR-Indian Veterinary Research Institute, Izatnagar, \\ Bareilly-243122- India
}

*Corresponding author

\section{Keywords}

Garlic, Turmeric,

Broiler, Growth

performance

Article Info

Accepted:

15 January 2018

Available Online:

10 February 2018

\section{A B S T R A C T}

A total of 120 , day-old broiler chicks were divided randomly into 4 treatment (CRD) groups with 3 replicates each i.e. 10 broiler chicks per replicate. Chicks of treatment $\mathrm{T}_{1}$ (control) were fed basal diet without any supplementation. In the treatment group $\mathrm{T}_{2}, \mathrm{~T}_{3}$ and $\mathrm{T}_{4}$, basal diet was supplemented with $3 \%$ garlic powder, $0.5 \%$ turmeric powder and $1.5 \%$ garlic powder plus $0.25 \%$ turmeric powder, respectively. The feeding trial lasted for 6 weeks viz., 0-3 weeks (starter phase) and 3-6 weeks (finisher phase). Body weight of individual chicks and feed intake of each replicate were recorded weekly for 6 weeks and growth performance of broilers was compared between the groups weekly. During $1^{\text {st }}$ week, significant reduction in feed intake of broilers of $T_{2}, T_{3}$ and $T_{4}$ as compared to $T_{1}$. During $2^{\text {nd }}$ week there was significant improvement in FCR only in broilers of $\mathrm{T}_{2}$. During $3^{\text {rd }}$ week, weight gain, FCR and PI were improved significantly in $T_{2}$ and $T_{4}$. In $4^{\text {th }}$ week, weight gain, FCR and PI of broilers of $\mathrm{T}_{2}$ and FCR and PI of $\mathrm{T}_{4}$ improved significantly. In $5^{\text {th }}$ week, there was significant improvement in weight gain, feed intake, FCR, PI and PER of $T_{2}$ broilers as compared to $T_{1}, T_{2}$ and $T_{3}$. Similarly, during $6^{\text {th }}$ week, significant improvement in weight gain, feed intake, FCR, PI and PER of $\mathrm{T}_{2}$ broilers. Also, in $\mathrm{T}_{4}$ broilers feed intake, weight gain and performance index increased significantly. In conclusion, 3\% garlic powder supplementation to broiler chicks enhanced growth performance.

\section{Introduction}

A variety of feed additives are being included in poultry diet to derive maximum growth of broiler chickens. Over the years, antibiotics are used as growth promoters in animal feeds. Use of in-feed-antibiotics leads to residues in meat and eggs and develops antibiotic resistance in human being consuming the poultry meat and eggs. Recently, many countries tended to prohibit antibiotics because of their side effects on both birds and human. Removing these kinds of growth promoters from broilers diet result in low 
growth performance, and also less resistance against diseases. To overcome the poor performance and the increased susceptibility to diseases, attempts have been made to find other alternatives.

Garlic (Allium sativum), a member of allium family (liliaceace) is a well known spice. In poultry nutrition, garlic is known to result in improved growth, inhibition of growth of pathogens in the gut, enhanced pancreatic function, and improved meat and carcass quality. Garlic can be used as a feed additive in broiler diets as it improves weight gain and feed conversion ratio (Mahmood et al., 2009). This performance improving property is attributed to the antibacterial properties of allicin and ajoene. Supplementation of garlic meal was reported to improve proliferation of absorptive cells in the gut. Dietary garlic supplementation increased villus height, crypt depth and ratio of villus height to crypt depth (Adibmoradi et al., 2006). Yason et al. (1987) stated that the crypt can be regarded as the villus factory, and a large crypt indicates fast tissue turnover and a high demand for new tissue. An improvement in either villus height or crypt depth may lead to an increase in nutrient absorption and better utility. Turmeric (Curcuma longa), a medicinal plant is a rhizomatous herbaceous perennial plant of ginger family, Zingiberaceae. Curcumin (diferuloylmethane) is the main yellow bioactive component of turmeric that has a wide spectrum of biological actions, including antioxidant, antibacterial, antifungal, antiprotozoal, antiviral, antiinflammatory, antihypertensive, and hypocholesteremic activities (Chattopadhyay et al., 2004). Other bioactive compounds also exhibit beneficial effects, such as demethoxy curcumin (antioxidant), bisdemethoxy curcumin (antioxidant), and sodium curcuminate (antiinflammatory). Turmeric is also used in gastrointestinal and respiratory disorders. Furthermore, it is used predominantly for internal and external injuries and has a protective effect on aflatoxin-induced mutagenicity. These significant biological properties of turmeric powder make it a potential substitute for infeed antibiotics in livestock diets. Turmeric is well known to have a property as a safe, natural, and residue free phytobiotics (Wang et al., 1998). Keeping the above facts in view, the present study was proposed with an aim to formulate a better herbal feed additive with turmeric and garlic for broiler birds to improve growth performance.

\section{Materials and Methods}

A feeding trial was conducted on day old 120 , broiler chicks in a completely randomised design (CRD). Garlic and turmeric powders were incorporated in a basal diet for a period of 42 days starting from day- old stage. Basal feeds were procured separately for starter (0-3 weeks) and finisher (4-6 weeks) phases of growth to meet the requirements of all the essential nutrients for growing broiler chicken as per BIS specification (1997). Physical and chemical composition of feed is given in Table 1. There were four dietary treatment groups $\left(T_{1}, T_{2}, T_{3}\right.$ and $\left.T_{4}\right)$ each with three replicates with ten birds in each replicate. Garlic powder, turmeric powder and its combination were supplemented in the broilers basal diet for a period of 6 weeks. Treatment $\mathrm{T}_{1}$ served as control in which broiler chicks were offered basal diet without any supplementation, treatment $\mathrm{T}_{2}$ was supplemented with $3 \%$ garlic powder, treatment $\mathrm{T}_{3}$ was supplemented with $0.5 \%$ turmeric powder and treatment $\mathrm{T}_{4}$ was supplemented with $1.5 \%$ garlic $+0.25 \%$ turmeric powders. The experimental feeds and water were provided ad libitum. For the study of growth performance, body weight of individual chick and feed consumption of each replicate group was recorded weekly upto $6^{\text {th }}$ week of age. Then, feed conversion 
ratio (FCR), performance index (PI) and protein efficiency ratio (PER) were calculated.

\section{Results and Discussion}

\section{$1^{\text {st }}$ week}

Feed intake of broilers in $\mathrm{T}_{2}(134.52 \mathrm{~g}), \mathrm{T}_{3}$ $(132.60 \mathrm{~g})$ and $\mathrm{T}_{4}(134.13 \mathrm{~g})$ groups was significantly $(\mathrm{P}<0.05)$ lowered than to $\mathrm{T}_{1}$ (138.97 g). Average body weight, body weight gain, feed conversion ratio (FCR), performance index and protein efficiency ratio (PER) showed no significant difference among the broilers of different treatment groups due to dietary supplementation of garlic and turmeric powder during the $1^{\text {st }}$ week of feeding trial.

\section{$2^{\text {nd }}$ week}

No significant difference $(\mathrm{P}>0.05)$ was observed in feed intake, average body weight, weight gain, performance index (PI) and protein efficiency ratio (PER) among broilers of different treatment groups during $2^{\text {nd }}$ week of feeding trial. FCR was improved in broilers of treatment $T_{2}$ (1.613) fed garlic powder supplemented diet compared to other treatment groups $\left(\mathrm{T}_{1}, \mathrm{~T}_{3}\right.$ and $\left.\mathrm{T}_{4}\right)$.

\section{$3^{\text {rd }}$ week}

During $3^{\text {rd }}$ week of feeding trial, feed intake and protein efficiency ratio was statistically similar among different treatment group of broilers. Body weight of broilers of garlic powder supplemented group $\mathrm{T}_{2}(540.50 \mathrm{~g})$ was significantly $(\mathrm{P}<0.05)$ increased as compared to other treatment groups $\mathrm{T}_{1}$ $(533.49 \mathrm{~g}), \mathrm{T}_{3}(534.67 \mathrm{~g})$ and $\mathrm{T}_{4}(536.41 \mathrm{~g})$. The body weight gain and FCR of broilers during 15-21 days was significantly $(\mathrm{P}<0.05)$ higher in garlic (254.23 g) and garlic plus turmeric powder $(252.83 \mathrm{~g})$ supplemented group as compared to control group (247.92 $\mathrm{g})$ and it was $251.16 \mathrm{~g}$ in turmeric supplemented group. Broilers of treatment $\mathrm{T}_{2}$ (152.73) group showed highest performance index during this period followed by $\mathrm{T}_{4}$ (151.44), $\mathrm{T}_{3}$ (149.89) and $\mathrm{T}_{1}(146.49)$ groups of broilers.

$4^{\text {th }}$ week

During this period, feed intake and protein efficiency ratio of broilers of different treatment groups was statistically $(\mathrm{P}>0.05)$ similar. Highest body weight of broiler chicks was recorded in treatment group $\mathrm{T}_{2}(848.96 \mathrm{~g})$ followed by $\mathrm{T}_{4}(842.29 \mathrm{~g}), \mathrm{T}_{3}(835.77 \mathrm{~g})$ and $\mathrm{T}_{1} \quad$ (834.21g). There was significantly $(\mathrm{P}<0.05)$ higher weight gain in broiler chicks of treatment $\mathrm{T}_{2}(308.46 \mathrm{~g})$ than other treatment groups 300.72, 301.10 and 305.89, respectively, in $\mathrm{T}_{1}, \mathrm{~T}_{3}$ and $\mathrm{T}_{4}$. However, there was significant $(\mathrm{P}<0.05)$ improvement in FCR and Performance index of broilers of treatment $\mathrm{T}_{2}$ and $\mathrm{T}_{4}$ as compared to treatments $\mathrm{T}_{3}$ and $\mathrm{T}_{1}$.

\section{$5^{\text {th }}$ week}

Maximum feed intake was recorded in broilers of treatment $\mathrm{T}_{2}$ and was significantly $(\mathrm{P}<0.05)$ higher than treatment groups $\mathrm{T}_{1}, \mathrm{~T}_{3}$ and statistically similar with $\mathrm{T}_{4}$. Body weight of broilers of treatment groups $\mathrm{T}_{2}$ and $\mathrm{T}_{4}$ showed significant $(\mathrm{P}<0.05)$ improvement as compared to control group $\mathrm{T}_{1}$. Significantly $(\mathrm{P}<0.05)$ higher weight gain and performance index was recorded in treatment $\mathrm{T}_{2}$ as compared to other treatments groups viz., $\mathrm{T}_{1}$, $\mathrm{T}_{3}$ and $\mathrm{T}_{4}$. There was significant $(\mathrm{P}<0.05)$ improvement in FCR and protein efficiency ratio of broilers of treatment group $\mathrm{T}_{2}$ (3.085) as compared to other treatment groups.

\section{$6^{\text {th }}$ week}

Feed intake, body weight and performance index of broilers of treatment $\mathrm{T}_{2}$ and $\mathrm{T}_{4}$ was significantly $(\mathrm{P}<0.05)$ higher than that of 
treatment $\mathrm{T}_{1}$ whereas feed intake, body weight and performance index of broilers of treatment $\mathrm{T}_{3}$ was statistically similar with control group $\mathrm{T}_{1}$. FCR also improved significantly $(\mathrm{P}<0.05)$ in garlic powder supplemented group $\mathrm{T}_{2}$ as compared to other treatments groups. During 36-42 days maximum weight gain was observed in $T_{2}$
$(447.72 \mathrm{~g})$ and was higher $(\mathrm{P}<0.05)$ as compared to weight gain $\mathrm{T}_{1}, \mathrm{~T}_{3}$ and comparable with $\mathrm{T}_{4}$. Protein efficiency ratio of broilers of garlic powder supplemented treatment group $\mathrm{T}_{2}$ (3.013) was significantly $(\mathrm{P}<0.05)$ better than other treatment groups $\mathrm{T}_{1}$ (3.048), $\mathrm{T}_{3}(3.053)$ and $\mathrm{T}_{4}(3.038)$.

Table.1 Ingredient and chemical composition (\%) of the basal diets

\begin{tabular}{|c|c|c|}
\hline Feed ingredients & $\begin{array}{c}\text { Broiler starter diet } \\
\text { (0-3 wks) }\end{array}$ & $\begin{array}{l}\text { Broiler finisher diet } \\
\text { (4-6 wks) }\end{array}$ \\
\hline Maize & 55.00 & 60.00 \\
\hline Deoiled soyabean meal & 36.00 & 32.00 \\
\hline Rice polish & 4.60 & 3.10 \\
\hline Soyabean oil & 0.50 & 1.00 \\
\hline Marble stone & 1.00 & 1.00 \\
\hline Dicalcium phosphate & 2.00 & 2.00 \\
\hline DL- Methionine & 0.10 & 0.10 \\
\hline Coccidiostat (Meduramycin) & 0.05 & 0.05 \\
\hline Copper sulphate & 0.01 & 0.01 \\
\hline Common salt & 0.30 & 0.30 \\
\hline Merivite -100 (Vitamin B12) & 0.02 & 0.02 \\
\hline Phosphoric acid & 0.10 & 0.10 \\
\hline Lipocare (choline chloride) & 0.05 & 0.05 \\
\hline Hepatocare & 0.10 & 0.10 \\
\hline Vitamin mixture & 0.03 & 0.03 \\
\hline Trace minerals & 0.14 & 0.14 \\
\hline \multicolumn{3}{|l|}{ Chemical Composition } \\
\hline Moisture & 9.20 & 10.90 \\
\hline Crude protein & 22.16 & 20.81 \\
\hline Crude fibre & 4.00 & 4.00 \\
\hline Ether extract & 4.50 & 4.25 \\
\hline $\begin{array}{l}\text { Metabolizable energy (kcal/kg) } \\
\text { calculated }\end{array}$ & 2877.00 & 2946.00 \\
\hline Nitrogen- free extract & 62.54 & 64.44 \\
\hline Total Ash & 6.80 & 6.50 \\
\hline Acid insoluble ash & 1.35 & 1.30 \\
\hline Calcium & 1.24 & 1.21 \\
\hline Phosphorus & 0.79 & 0.77 \\
\hline
\end{tabular}


Table.2 Average growth performance of broiler chicks from 0-7 days fed diets supplemented with garlic and turmeric powder

\begin{tabular}{|c|c|c|c|c|}
\hline \multirow[t]{3}{*}{ Parameters } & \multicolumn{4}{|c|}{ Treatments/ Groups } \\
\hline & $\mathrm{T}_{1}$ & $\mathrm{~T}_{2}$ & $\mathrm{~T}_{3}$ & $\mathrm{~T}_{4}$ \\
\hline & Control & Garlic (3\%) & $\begin{array}{l}\text { Turmeric } \\
(0.5 \%)\end{array}$ & $\begin{array}{c}\text { Garlic }(1.5 \%)+ \\
\text { Turmeric } \\
(0.25 \%)\end{array}$ \\
\hline $\begin{array}{l}\text { Initial body } \\
\text { weight (g) }\end{array}$ & $41.20 \pm 0.15$ & $41.23 \pm 0.03$ & $41.20 \pm 0.05$ & $41.20 \pm 0.11$ \\
\hline $\begin{array}{c}\text { Body weight at } \\
7^{\text {th }} \text { day (g) }\end{array}$ & $135.13 \pm 0.70$ & $132.77 \pm 1.39$ & $131.97 \pm 0.94$ & $132.93 \pm 1.48$ \\
\hline $\begin{array}{c}\text { Weight gain } \\
\text { (g) }\end{array}$ & $93.93 \pm 0.83$ & $91.53 \pm 1.37$ & $90.77 \pm 0.92$ & $91.73 \pm 1.36$ \\
\hline $\begin{array}{l}\text { Feed intake } \\
\qquad(\mathrm{g}) *\end{array}$ & $138.97^{\mathrm{a}} \pm 0.59$ & $134.52^{b} \pm 0.69$ & $132.60^{b} \pm 1.10$ & $134.13^{b} \pm 2.23$ \\
\hline $\begin{array}{c}\text { Feed } \\
\text { conversion } \\
\text { ratio }\end{array}$ & $1.480 \pm 0.006$ & $1.470 \pm 0.016$ & $1.461 \pm 0.021$ & $1.462 \pm 0.011$ \\
\hline $\begin{array}{c}\text { Performance } \\
\text { index }\end{array}$ & $63.50 \pm 0.85$ & $62.30 \pm 1.58$ & $62.16 \pm 1.44$ & $62.74 \pm 1.06$ \\
\hline $\begin{array}{c}\text { Protein } \\
\text { efficiency ratio }\end{array}$ & $3.376 \pm 0.015$ & $3.377 \pm 0.036$ & $3.420 \pm 0.048$ & $3.410 \pm 0.026$ \\
\hline
\end{tabular}

Table.3 Average growth performance of broiler chicks from 8-14 days fed diets supplemented with garlic and turmeric powder

\begin{tabular}{|c|c|c|c|c|}
\hline \multirow[t]{3}{*}{ Parameters } & \multicolumn{4}{|c|}{ Treatments/ Groups } \\
\hline & $\mathrm{T}_{1}$ & $\mathrm{~T}_{2}$ & $\mathrm{~T}_{3}$ & $\mathrm{~T}_{4}$ \\
\hline & Control & Garlic (3\%) & Turmeric $(0.5 \%)$ & $\begin{array}{c}\text { Garlic }(1.5 \%)+ \\
\text { Turmeric }(0.25 \%)\end{array}$ \\
\hline $\begin{array}{c}\text { Body weight at } 14^{\text {th }} \\
\text { day }(\mathrm{g})\end{array}$ & $285.57 \pm 0.77$ & $286.27 \pm 1.16$ & $282.51 \pm 0.56$ & $283.58 \pm 0.69$ \\
\hline Weight gain (g) & $150.43 \pm 0.67$ & $153.50 \pm 0.64$ & $150.55 \pm 0.95$ & $150.65 \pm 1.18$ \\
\hline Feed intake (g) & $245.53 \pm 0.71$ & $247.67 \pm 0.75$ & $246.30 \pm 0.66$ & $245.00 \pm 1.27$ \\
\hline $\begin{array}{c}\text { Feed conversion } \\
\text { ratio* }\end{array}$ & $1.632^{\mathrm{a}} \pm 0.002$ & $1.613^{b} \pm 0.001$ & $1.636^{\mathrm{a}} \pm 0.005$ & $1.626^{\mathrm{ab}} \pm 0.005$ \\
\hline Performance index* & $92.17 \pm 0.56$ & $95.14 \pm 0.51$ & $92.02 \pm 0.92$ & $92.64 \pm 1.00$ \\
\hline $\begin{array}{c}\text { Protein efficiency } \\
\text { ratio }\end{array}$ & $3.060 \pm 0.004$ & $3.076 \pm 0.003$ & $3.053 \pm 0.011$ & $3.067 \pm 0.009$ \\
\hline
\end{tabular}


Table.4 Average growth performance of broiler chicks from 15-21 days fed diets supplemented with garlic and turmeric powder

\begin{tabular}{|c|c|c|c|c|}
\hline \multirow{2}{*}{ Parameters } & \multicolumn{4}{|c|}{ Treatments/ Groups } \\
\cline { 3 - 5 } & $\mathrm{T}_{1}$ & $\mathrm{~T}_{2}$ & $\mathrm{~T}_{3}$ & $\mathrm{~T}_{4}$ \\
\cline { 3 - 5 } & Control & Garlic $(3 \%)$ & Turmeric $(0.5 \%)$ & $\begin{array}{c}\text { Garlic }(1.5 \%)+ \\
\text { Turmeric } \\
(0.25 \%)\end{array}$ \\
\hline $\begin{array}{c}\text { Body weight at } \\
\mathbf{2 1}^{\text {st }} \text { day (g)* }\end{array}$ & $533.49^{\mathrm{b}} \pm 0.69$ & $540.50^{\mathrm{a}} \pm 0.82$ & $534.67^{\mathrm{b}} \pm 1.0$ & $536.41^{\mathrm{b}} \pm 1.54$ \\
\hline $\begin{array}{c}\text { Weight gain } \\
\text { (g)* }\end{array}$ & $247.92^{\mathrm{b}} \pm 0.93$ & $254.23^{\mathrm{a}} \pm 1.08$ & $251.16^{\mathrm{ab}} \pm 1.52$ & $252.83^{\mathrm{a}} \pm 0.90$ \\
\hline $\begin{array}{c}\text { Feed intake } \\
\text { (g) }\end{array}$ & $419.57 \pm 1.03$ & $423.20 \pm 0.65$ & $420.87 \pm 1.20$ & $422.10 \pm 0.40$ \\
\hline $\begin{array}{c}\text { Feed } \\
\text { conversion } \\
\text { ratio* }\end{array}$ & $1.692^{\mathrm{a}} \pm 0.002$ & $1.665^{\mathrm{bc}} \pm 0.006$ & $1.675^{\mathrm{ab}} \pm 0.006$ & $1.669^{\mathrm{b}} \pm 0.005$ \\
\hline $\begin{array}{c}\text { Performance } \\
\text { index* }\end{array}$ & $146.49^{\mathrm{b}} \pm 0.74$ & $152.73^{\mathrm{a}} \pm 1.21$ & $149.89^{\mathrm{ab}} \pm 1.47$ & $151.44^{\mathrm{a}} \pm 1.07$ \\
\hline $\begin{array}{c}\text { Protein } \\
\text { efficiency ratio }\end{array}$ & $2.952 \pm 0.004$ & $2.981 \pm 0.011$ & $2.981 \pm 0.011$ & $2.987 \pm 0.010$ \\
\hline $\begin{array}{c}\mathrm{a}, \mathrm{b}, \mathrm{c} \\
\text { values bearing different superscripts in a row differ significantly } * \mathrm{P}<0.05\end{array}$ \\
\hline
\end{tabular}

Table.5 Average growth performance of broiler chicks from 22-28 days fed diets supplemented with garlic and turmeric powder

\begin{tabular}{|c|c|c|c|c|}
\hline \multirow[t]{3}{*}{ Parameters } & \multicolumn{4}{|c|}{ Treatments/ Groups } \\
\hline & $\mathrm{T}_{1}$ & $\mathrm{~T}_{2}$ & $\mathrm{~T}_{3}$ & $\mathrm{~T}_{4}$ \\
\hline & Control & Garlic (3\%) & Turmeric $(0.5 \%)$ & $\begin{array}{c}\text { Garlic }(1.5 \%)+ \\
\text { Turmeric }(0.25 \%)\end{array}$ \\
\hline $\begin{array}{l}\text { Body weight at } 28^{\text {th }} \\
\text { day }(g)^{*}\end{array}$ & $834.21^{c} \pm 2.31$ & $848.96^{\mathrm{a}} \pm 1.12$ & $835.77^{c} \pm 0.93$ & $842.29^{\mathrm{b}} \pm 1.42$ \\
\hline Weight gain $(g)^{*}$ & $300.72^{b} \pm 1.62$ & $308.46^{\mathrm{a}} \pm 1.89$ & $301.10^{b} \pm 1.51$ & $305.89^{\mathrm{ab}} \pm 1.39$ \\
\hline Feed intake (g) & $521.20 \pm 0.91$ & $523.63 \pm 2.54$ & $521.60 \pm 1.04$ & $522.47 \pm 0.63$ \\
\hline $\begin{array}{c}\text { Feed conversion } \\
\text { ratio* }\end{array}$ & $1.733^{\mathrm{a}} \pm 0.006$ & $1.698^{b} \pm 0.004$ & $1.732^{\mathrm{a}} \pm 0.005$ & $1.708^{b} \pm 0.008$ \\
\hline Performance index* & $173.51^{b} \pm 1.59$ & $181.71^{\mathrm{a}} \pm 1.47$ & $173.82^{b} \pm 1.39$ & $179.10^{\mathrm{a}} \pm 1.70$ \\
\hline $\begin{array}{c}\text { Protein efficiency } \\
\text { ratio }\end{array}$ & $3.149 \pm 0.0111$ & $3.147 \pm 0.008$ & $3.154 \pm 0.009$ & $3.165 \pm 0.015$ \\
\hline
\end{tabular}


Table.6 Average growth performance of broiler chicks from 29-35days fed diets supplemented with garlic and turmeric powder

\begin{tabular}{|c|c|c|c|c|}
\hline \multirow[t]{3}{*}{ Parameters } & \multicolumn{4}{|c|}{ Treatments/ Groups } \\
\hline & $\mathrm{T}_{1}$ & $\mathrm{~T}_{2}$ & $\mathrm{~T}_{3}$ & $\mathrm{~T}_{4}$ \\
\hline & Control & Garlic (3\%) & $\begin{array}{c}\text { Turmeric } \\
(0.5 \%)\end{array}$ & $\begin{array}{c}\text { Garlic }(1.5 \%)+ \\
\text { Turmeric } \\
(0.25 \%)\end{array}$ \\
\hline Body weight at $35^{\text {th }}$ day $(\mathrm{g})^{*}$ & $1253.02^{\mathrm{c}} \pm 1.92$ & $1276.70^{\mathrm{a}} \pm 2.32$ & $1255.75^{\mathrm{c}} \pm 0.47$ & $1264.75^{\mathrm{b}} \pm 0.30$ \\
\hline $\begin{array}{c}\text { Weight gain } \\
(\mathrm{g})^{*}\end{array}$ & $418.81^{b} \pm 1.67$ & $427.73^{\mathrm{a}} \pm 1.27$ & $420.98^{b} \pm 1.31$ & $422.45^{b} \pm 1.31$ \\
\hline Feed intake $(\mathrm{g}) *$ & $734.80^{b} \pm 1.36$ & $740.63^{\mathrm{a}} \pm 1.13$ & $736.57^{\mathrm{b}} \pm 0.82$ & $737.63^{\mathrm{ab}} \pm 1.27$ \\
\hline Feed conversion ratio* & $1.754^{\mathrm{a}} \pm 0.004$ & $1.732^{\mathrm{b}} \pm 0.002$ & $1.750^{\mathrm{a}} \pm 0.003$ & $1.746^{\mathrm{a}} \pm 0.003$ \\
\hline Performance index* & $238.71^{b} \pm 1.48$ & $247.03^{\mathrm{a}} \pm 1.12$ & $240.61^{\mathrm{b}} \pm 1.27$ & $241.94^{\mathrm{b}} \pm 1.24$ \\
\hline Protein efficiency ratio* & $3.111^{\mathrm{ab}} \pm 0.007$ & $3.085^{\mathrm{c}} \pm 0.005$ & $3.123^{\mathrm{a}} \pm 0.006$ & $3.096^{\mathrm{bc}} \pm 0.006$ \\
\hline
\end{tabular}

Table.7 Average growth performance of broiler chicks from 36-42 days fed diets supplemented with garlic and turmeric powder

\begin{tabular}{|c|c|c|c|c|}
\hline \multirow[t]{3}{*}{ Parameters } & \multicolumn{4}{|c|}{ Treatments/ Groups } \\
\hline & $\mathrm{T}_{1}$ & $\mathrm{~T}_{2}$ & $\mathrm{~T}_{3}$ & $\mathrm{~T}_{4}$ \\
\hline & Control & Garlic (3\%) & Turmeric $(0.5 \%)$ & $\begin{array}{c}\text { Garlic }(1.5 \%)+ \\
\text { Turmeric }(0.25 \%)\end{array}$ \\
\hline $\begin{array}{l}\text { Body weight 42th day } \\
\text { (g) }\end{array}$ & $1694.11^{c} \pm 0.70$ & $1724.41^{\mathrm{a}} \pm 3.31$ & $1697.54^{c} \pm 1.59$ & $1710.12^{\mathrm{b}} \pm 1.04$ \\
\hline Weight gain $(\mathrm{g}) *$ & $441.08^{b} \pm 1.24$ & $447.72^{\mathrm{a}} \pm 1.04$ & $441.79^{b} \pm 1.15$ & $445.37^{\mathrm{ab}} \pm 1.12$ \\
\hline Feed intake $(\mathrm{g})^{*}$ & $790.00^{c} \pm 0.38$ & $793.87^{\mathrm{a}} \pm 0.76$ & $790.77^{\mathrm{bc}} \pm 0.94$ & $792.53^{\mathrm{ab}} \pm 0.32$ \\
\hline $\begin{array}{c}\text { Feed conversion } \\
\text { ratio }\end{array}$ & $1.791^{\mathrm{a}} \pm 0.004$ & $1.773^{\mathrm{bc}} \pm 0.002$ & $1.793^{\mathrm{a}} \pm 0.003$ & $1.780^{\mathrm{b}} \pm 0.003$ \\
\hline Performance index & $246.27^{c} \pm 1.27$ & $252.50^{\mathrm{a}} \pm 0.94$ & $246.82^{b c} \pm 1.33$ & $250.28^{\mathrm{ab}} \pm 1.17$ \\
\hline $\begin{array}{c}\text { Protein efficiency } \\
\text { ratio* }\end{array}$ & $3.048^{\mathrm{a}} \pm 0.007$ & $3.013^{\mathrm{b}} \pm 0.004$ & $3.053^{\mathrm{a}} \pm 0.006$ & $3.038^{\mathrm{a}} \pm 0.006$ \\
\hline
\end{tabular}

During 0-7 days there was significantly less feed intake in broilers due to garlic and turmeric powder supplementation in diet which might be due to repulsive odour and taste of garlic and turmeric powder. The increase in growth performance in broilers due to feeding of garlic powder supplemented diet might be attributed to allicin, component 
of garlic which promotes the performance of intestinal flora, thereby improving digestion and utilization of nutrients by increasing villi length and width of intestine leading to improved growth performance (Lewis et al., 2003). The growth performance of broilers fed turmeric supplemented diet did not show significant difference from control group. Performance of garlic plus turmeric powder supplemented group of broilers significantly increased as compared to control group of broilers which might be due to growth promoting effect of garlic.

The present results indicated that dietary garlic powder supplementation significantly improved growth performance and are in agreement with the findings of Pourali et al., (2010), Onyimonyi et al., (2011) and Oladele et al., (2012) who reported that garlic powder has positive effects on growth performance. Abou-Elkhair et al., (2014) and Akbarian et al., (2012) who reported that growth performance of broilers was not influenced by inclusion of turmeric in the diet. On the contrary, Raghdad (2012) and Swathi et al., (2012) reported that addition of turmeric powder in the diet of broilers had significant improvement in body weight and feed conversion ratio.

From the present investigation it can be concluded that supplementation of garlic @ $30 \mathrm{~g} / \mathrm{kg}$ of feed of broiler chicks improved growth performance whereas turmeric powder supplementation @ 5g/kg of feed of broiler chicks did not altered growth performance.

\section{References}

Abou-Elkhair, R., Ahmed, H. A. and Selim. S. 2014. Effect of black pepper (Piper nigrum), turmeric powder (Curcuma longa) and coriander seeds (Coriander sativum) and their combination as feed additives on growth performance, carcass traits, some blood parameters and humoral immune response of broiler chickens. Asian Australasian Journal of Animal Sciences. 27 (6): 847-54.

Adibmoradi, M., Navidshad, B., Seifdavati, J., and Royan, M. 2006. Effect of dietary garlic meal on histological structure of small intestine in broiler chickens. The Journal of Poultry Science. 43: 378-83.

Akbarian, A., Golian, A., Kermanshahi, H., Gilani, A. and Moradi, S. 2012. Influence of turmeric rhizome and black pepper on blood constituents and performance of broiler chickens. African Journal of Biotechnology. 11 (34): 8606-11.

BIS. 1997. Poultry feeds- Specification, IS1374. Bureau of Indian Standards, Manak Bhawan, New Delhi, India.

Chattopadhyay, I., Biswas, K., Bandyopadhyay, U. and Banerjee, R. K. 2004. Turmeric and curcumin: Biological actions and medicinal applications. Current Science .87: 4453.

Lewis, M. R., Rose, S. P., Mackenzie, A. M. and Tucker, L. A. 2003. Effects of dietary inclusion of plant extracts on the growth performance of male broiler chickens. British Poultry Science. 44: 43-44.

Mahmood, S., Mushtaq-Ul-Hassan, M., Alam, M., and Ahmad, F. 2009. Comparative efficacy of Nigella sativa and Allium sativum as growth promoters in broilers. International Journal of Agriculture and Biology. 11: 775-78.

Oladele, O. A., Emikpe, B. O. and Bakare, H. 2012. Effects of dietary garlic (Allium sativum) supplementation on body weight and gut morphometry of commercial broilers. International Journal of Morphology. 30 (1): 238.

Onyimonyi, A. E., Chukwuma, P. C. and 
Igbokwe, C. 2011. Growth and hypocholesterolemic properties of dry garlic powder (Allium sativum) on broilers. African Journal of Biotechnology. 11(11): 2666-71.

Pourali, M., Mirghelenj, S. A. and Kermanshahi, H. 2010. Effect of garlic powder on production performance and immune response of broiler chickens challenged with Newcastle disease virus. Global Veterinaria. 4 (6): 61621.

Raghdad, A. and Al-Jaleel, A. 2012. Use of turmeric (Curcuma longa) on the performance and some physiological traits on the broiler diets. The Iraqi Journal of Veterinary Medicine. 36 (1): 51-57.

Swathi, B., Gupta, P.S.P. and Nagalaxhmi, P.
2012. Effect of tulsi and turmeric on broiler performance and blood constituents during heat stress in broilers. International Journal of Pharmacy and Biological Science. 3(3): 446-53.

Wang, R., Li. D. and Bourne, S. 1998. Can 2000 years of herbal medicine history help us solve problems in the year 2000? In: Biotechnology in the Feed Industry. Proceedings of Alltech's $14^{\text {th }}$ Annual Symposium, ed. Lyons TP and Jacques KA. pp. 273-91.

Yason, C. V., Summers, B. A., and Schat, K. A. 1987. Pathogenesis of rotavirus infection in various age groups of chickens and turkeys. American Journal of Veterinary Research. 48: 927-38.

\section{How to cite this article:}

Khwairakpam Ratika, R.K. James Singh, Ram Kumar Singh and Mala Singh. 2018. Weekly assessment of growth performance of broilers fed diet supplemented with garlic and turmeric powder and their combination. Int.J.Curr.Microbiol.App.Sci. 7(02): 1373-1381. doi: https://doi.org/10.20546/ijcmas.2018.702.166 